\title{
-NOTES-
}

\section{A PROBLEM OF THE TEMPERATURE DISTRIBUTION IN A MOVING MEDIUM*}

\section{By A. E. BENFIELD (Cruft Laboratory, Harvard University)}

The thermal problem solved in this paper is primarily of geophysical interest. However, it seems worth while to give an account of it here, as the boundary conditions are rather unusual and the solution, which makes use of the Laplace method, involves a transform not given in the usual references. It is thought that the problem may be of interest to those generally concerned with questions of thermal conductivity. The geophysical implications will be discussed in another paper, to be published elsewhere. $\dagger$

We wish to find the temperature $T(x, t)$, where $x$ is the space coordinate and $t$ is time, in a semi-infinite medium, having initially a constant temperature gradient, $A$, when the medium moves to the left with a positive constant speed, $v$, as shown in

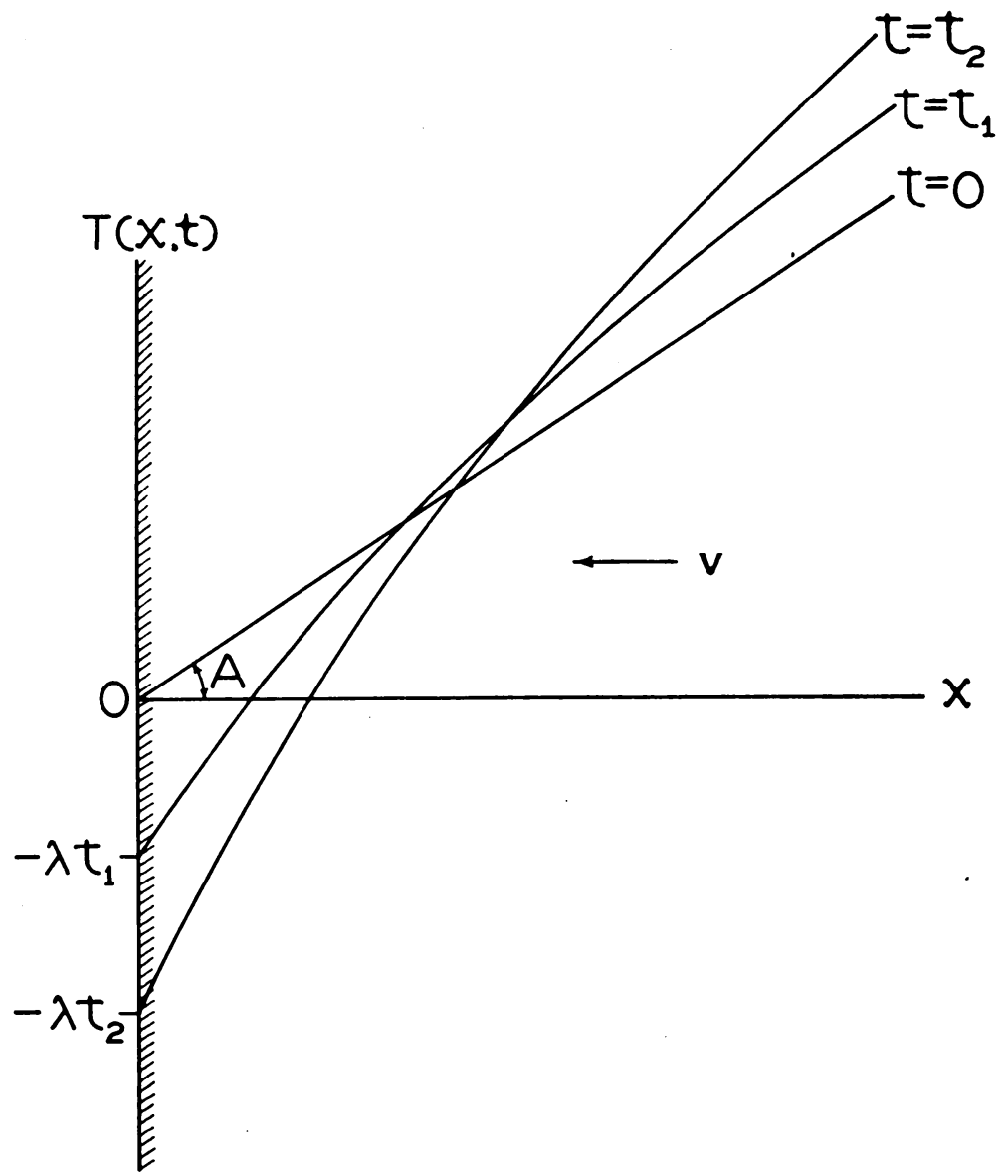

FIG. 1. Qualitative curves showing $T(x, t)$ at times $t=0, t_{1}, t_{2}=2 t_{1}$ where $0<t_{1}<t_{2} . T(0, t)=-\lambda t$.

*Received June 15, 1948.

†A. E. Benfield, J. Appl. Phys. (in press). 
Fig. 1. No material passes the boundary plane $x=0$, but instead, on reaching it, the material is annihilated without the evolution of heat. Furthermore, the plane $x=0$ is maintained at a temperature which decreases linearly with time at the rate $\lambda$. Thereforethe boundary conditions are as follows: when $t=0, T(x, 0)=A x$; and at $x=0, T(0, t)=$ $-\lambda t$, where $\lambda$ is a constant.

The application of the divergence theorem shows that the differential equation to be satisfied is

$$
K \frac{\partial^{2} T}{\partial x^{2}}+v \frac{\partial T}{\partial x}=\frac{\partial T}{\partial t}
$$

where $K$ is the thermal diffusivity. The medium moves in the negative $x$ direction with this sign convention the speed $v=-d x / d t>0$.
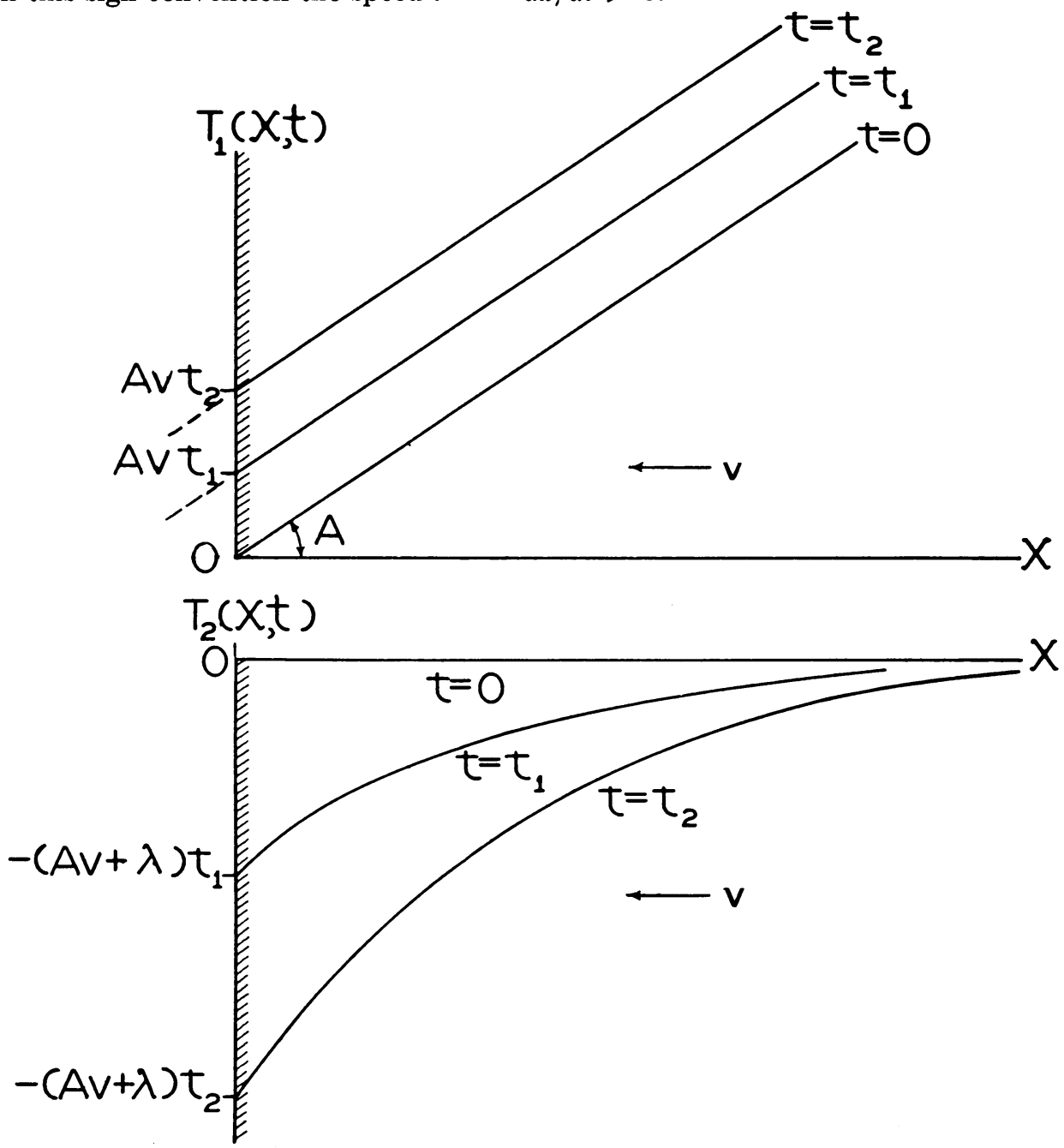

Fig. 2a. Curves for $T_{1}(x, t)=A(x+v t)$. $T_{1}(0, t)=A v t$.

FIg. 2b. Qualitative curves for $T_{2}(x, t) . T_{2}(0, t)=-(A v+\lambda) t$. $T(x, t)=T_{1}(x, t)+T_{2}(x, t)$. 
The problem may be split into two parts; thus

$$
T(x, t)=T_{1}(x, t)+T_{2}(x, t)
$$

where

$$
T_{1}(x, t)=A(x+v t)
$$

as shown in Fig. 2a, and $T_{2}$, which is shown qualitatively in Fig. 2b, remains to be found. The boundary conditions for $T_{2}(x, t)$, however, are not hard to find; by considering Eq. (2), and the boundary conditions for $T$ and $T_{1}$, we see that they must be $T_{2}(0, t)=$ $-(A v+\lambda) t$ and $T_{2}(x, 0)=0$. As $T_{1}(x, t)$ obviously satisfies Eq. (1), $T_{2}(x, t)$ must do the same.

Calling $\bar{T}_{2}$ the Laplace transform of $T_{2}$, defined by

$$
\bar{T}_{2}=L\left\{T_{2}\right\}=\int_{0}^{\infty} e^{-p t} T_{2}(x, t) d t,
$$

Eq. (1) becomes

$$
K \frac{d^{2} \bar{T}_{2}}{d x^{2}}+v \frac{d \bar{T}_{2}}{d x}-p \bar{T}_{2}=0
$$

where $p$, in Eqs. (4) and (5), is the usual constant, as used by Carslaw and Jaeger. ${ }^{1}$

The solution of Eq. (5), which is a second order differential equation with constant coefficients, is well-known and may be written as

$$
e^{-v x / 2 K}\left[B_{1} \exp \left\{x\left[v^{2} / 4 K^{2}+p / K\right]^{1 / 2}\right\}+B_{2} \exp \left\{-x\left[v^{2} / 4 K^{2}+p / K\right]^{1 / 2}\right\}\right]
$$

where $B_{1}$ and $B_{2}$ are the arbitrary constants, whose values depend on boundary conditions. However, as we are interested in a solution of $T_{2}$ which remains bounded, and in fact equals zero, as $x \rightarrow \infty$, we shall set $B_{1}=0$ and write

$$
\bar{T}_{2}=B_{2} e^{-v x / 2 K} \exp \left\{-x\left[v^{2} / 4 K^{2}+p / K\right]^{1 / 2}\right\} .
$$

We must now find the value of $B_{2}$, which is done in the conventional way by considering the situation when $x=0$, for which value of $x, \bar{T}_{2}=B_{2}$. Since $T_{2}(0, t)=-(A v+\lambda) t$, it follows that when $x=0, \bar{T}_{2}=B_{2}=-(A v+\lambda) L\{t\}$; and so, since $L\{t\}=1 / p^{2}$, we can now rewrite Eq. (6) as

$$
\bar{T}_{2}=-(A v+\lambda) e^{-v x / 2 K} \frac{\exp \left\{-x\left[v^{2} / 4 K^{2}+p / K\right]^{1 / 2}\right\}}{p^{2}} .
$$

In order to find $T_{2}$ we now need to know $L^{-1}\left[\exp \left\{-x\left[v^{2} / 4 K^{2}+p / K\right]^{1 / 2}\right\} / p^{2}\right]$. It is known ${ }^{2}$ that

$$
\begin{aligned}
L^{-1}\left[\frac{\exp \left\{-x[(\nu+p) / K]^{1 / 2}\right\}}{p}\right]=\frac{1}{2} & \exp \left\{+x(\nu / K)^{1 / 2}\right\} \operatorname{erfc}\left[\frac{x}{2(K t)^{1 / 2}}+(\nu t)^{1 / 2}\right] \\
& +\frac{1}{2} \exp \left\{-x(\nu / K)^{1 / 2}\right\} \operatorname{erfc}\left[\frac{x}{2(K t)^{1 / 2}}-(\nu t)^{1 / 2}\right]
\end{aligned}
$$

${ }^{1} \mathrm{H}$. S. Carslaw and J. C. Jaeger, Conduction of heat in solids, Clarendon Press, Oxford, 1947, ch. XI. ${ }^{2}$ Cf. Carslaw and Jaeger, loc. cit., p. 270, Eqs. (2) and (3). 
where erfc $y=1-2 \pi^{-1 / 2} \int_{0}^{y} e^{-u^{2}} d u$. If we set $\nu=v^{2} / 4 K$ and make use of the theorem ${ }^{3}$ that

$$
L\left\{\int_{0}^{t} T\left(t^{\prime}\right) d t^{\prime}\right\}=\frac{1}{p} L\{T\}
$$

it simply remains for us to integrate the right-hand side of Eq. (8) with respect to time. Related problems have been considered by Jaeger, ${ }^{4}$ Paterson $^{5}$ and Horenstein. ${ }^{6}$

Proceeding now to the integration we find, integrating by parts, that

$$
\int_{0}^{t} \operatorname{erfc} f(t) d t=t \operatorname{erfc} f+\frac{2}{\pi^{1 / 2}} \int_{\infty}^{f} t e^{-f^{2}} d f
$$

where $f(t)=a t^{-1 / 2}+b t^{1 / 2}, a=x / 2 K^{1 / 2}$ and $b=\nu^{1 / 2}=v / 2 K^{1 / 2}$. Writing $t=(1 / b)\left(t^{1 / 2} f-a\right)$, we find that

$$
\int_{\infty}^{f} t \cdot e^{-f^{2}} d f=\frac{a \pi^{1 / 2}}{2 b} \operatorname{erfc} f+\frac{1}{b} \int_{\infty}^{f} t^{1 / 2} f e^{-f^{2}} d f
$$

The integral $\int_{\infty}^{f} t^{1 / 2} f e^{-f^{2}} d f$ in Eq. (10) may be evaluated by integrating by parts and further substitutions, but for the case in hand this is unnecessary. This is because Eq. (8) contains not only the expression erfc $f$, but also erfc $g$ where $g(t)=a t^{-1 / 2}-b t^{1 / 2}$; and as a result of the symmetry of $f$ and $g$ it turns out that the second term of the righthand side of Eq. (10) cancels in the integration of Eq. (8).

Therefore, combining Eqs. (9) and (10), we find that integrating Eq. (8) leads to

$$
L^{-1}\left[\frac{\exp \left\{-x\left[\left(b^{2}+p\right) / K\right]^{1 / 2}\right\}}{p^{2}}\right]=\frac{1}{2} e^{2 a b}\left(t+\frac{a}{b}\right) \operatorname{erfc} f+\frac{1}{2} e^{-2 a b}\left(t-\frac{a}{b}\right) \operatorname{erfc} g
$$

from which it follows that

$$
T_{2}(x, t)=-\frac{1}{2}\left(A+\frac{\lambda}{v}\right)\left[(x+v t) \operatorname{erfc}\left(\frac{x+v t}{2(K t)^{1 / 2}}\right)-(x-v t) e^{-v x / K} \operatorname{erfc}\left(\frac{x-v t}{2(K t)^{1 / 2}}\right)\right] .
$$

It can be shown that Eq. (12) satisfies Eq. (1) and the boundary conditions for $T_{2}(x, t)$. Furthermore, one can demonstrate that if we allow $v \rightarrow \infty$, Eq. (12) leads to the appropriate expression for the temperature distribution in a stationary medium. ${ }^{7}$

The solution of our problem, therefore, is the sum of Eqs. (3) and (12). This is shown graphically in Fig. 1, which is the sum of Figs. 2a and $2 \mathrm{~b}$.

Equation (12) may be derived by other means than the method used here. For instance, one may use the substitution 8

$$
T_{2}=T^{*} e^{-v x / 2 K-v^{2} t / 4 K}
$$

and make use of the fact that $T^{*}$ satisfies the differential equation $K \partial^{2} T^{*} / \partial x^{2}=\partial T^{*} / \partial t$,

${ }^{3}$ Cf. Carslaw and Jaeger, loc. cit., p. 242, th. IV.

${ }^{4} J$. C. Jaeger, Quart. Appl. Math. 4, 100-103 (1946).

5S. Paterson, Quart. Appl. Math. 4, 305-306 (1946).

${ }^{6} \mathrm{~W}$. Horenstein, Quart. Appl. Math. 3, 183-184 (1945).

${ }^{7}$ Cf. Carslaw and Jaeger, loc. cit., p. 45, eq. (4).

${ }^{8}$ See, for instance, P. Frank and R. von Mises, Differentialgleichungen der Physik, F. Vieweg \& Sohn, Braunschweig, 1935, vol. 2, p. 605. 
thus transforming the problem into one of a stationary, rather than a moving, medium. However, on applying the new boundary conditions, for $T^{*}$, there results another untabulated Laplace transform. In this case, the required transform may be found by a differentiation, rather than an integration, but the differentiation seems to involve a step of doubtful justifiability.

In conclusion, I wish to thank Dr. P. E. LeCorbeiller for his interest in this problem and for several helpful discussions of it.

\title{
ON A CLASS OF SINGULAR INTEGRAL EQUATIONS OCCURRING IN PHYSICS*
}

\author{
By H. P. THIELMAN (Iowa State College)
}

1. Introduction. Certain boundary value problems ${ }^{1}$ in electrodynamics can be formulated mathematically as Wiener-Hopf ${ }^{2}$ integral equations. The equations are of the form

$$
f(x)=\int_{0}^{\infty} K(|x-y|) g(y) d y, \quad x>0
$$

where $K(|x-y|)$ is a real function of the absolute value of the difference between $x$ and $y$. The Eq. (1) is an integral equation of the "first kind", $K(|x-y|)$ and $f(x)$ are given functions, and $g(y)$ is unknown. One advantage of formulating a given problem, if possible, as a Wiener-Hopf integral equation is that such equations are susceptible to the application of the theory of Fourier transforms. The application of the latter theory is especially advantageous when the Fourier transforms of the given functions are easily found. The object of the present paper is to show that for a certain class of Wiener-Hopf integral equations a more direct method yields the explicit solution in a more elementary and simpler way. The present method applies, however, only to those integral equations of type (1), and to integral equations of the second kind such as

$$
f(x)=g(x)-\lambda \int_{0}^{\infty} K(|x-y|) g(y) d y, \quad x>0
$$

if $K(|x-y|)$ satisfies the same linear homogeneous differential equations with constant coefficients in each of the regions $0 \leq y \leq x$, and $0 \leq x \leq y$. It should be mentioned here that the special case of Eq. (2) with $f(x) \equiv 0$, and $K(|x-y|)=e^{-|x-y|}$ was treated by Lalesco ${ }^{3}$ by a method similar to the one given here.

2. The general theory. We consider an integral equation of the form

$$
f(x)=\lambda \int_{0}^{\infty} K(|x-y|) g(y) d y \quad x>0
$$

*Received May 11, 1948.

1J. F. Carlson and A. E. Heins, The reflection of an electromagnetic plane wave by an infinite set of plates. I, Q. Appl. Math. 4, 313-329 (1947).

${ }^{2}$ N. Wiener, E. Hopf, Über eine Klasse singulärer Integralgleichungen, Sitzungsber. Preuss. Ak. Wissensch. 696-706 (1931).

${ }^{3}$ Trajan Lalesco, Théorie des équations intégrales, A. Hermann et Fils, Paris, 1912, p. 121. 